\title{
THE USE OF UNIMATRIXR MATRIX SYSTEM FOR RESTORATION OF INTERDENTAL CONTACT POINTS - GASE REPORT
}

\section{Bărbuceanu Lucian-Codruț', Gheorghiu Irina-Maria², Perlea Paula2, Mitran Loredana3, Mitran Mihaĩ2, Iliescu Alexandru-Andre ${ }^{4}$}

${ }^{1}$ Dentist, Private dental Practice, Bucharest

${ }^{2}$ Faculty of Dental Medicine, UMF "Carol Davila" Bucharest

${ }^{3}$ Department of Otorhinolaryngology, Elias Hospital

${ }^{4}$ Faculty of Dental Medicine, UMF Craiova

Irina-Maria Gheorghiu

Faculty of Dental Medicine, UMF “Carol Davila” Bucharest Calea Plevnei 17-23, Bucuresti; email:igheorghiu@hotmail.com

\begin{abstract}
Proper restoration of proximal contact points is a great challenge for any dentist. The special particularities related to an appropriate anatomical and functional restoration at this level are related first of all to the knowledge of the local topography, to the possibilities of dental isolation, the type and the extension of the loss of hard dental substance. Currently, multiple techniques and specific conformation systems are available, which can be adapted to each clinical situation, so that the outcome of the therapeutic maneuver is a success.
\end{abstract}

Keywords: class II restoration; contact point; matrix system

\section{Introduction}

Proper restoration of interdental contact points is one of the most important challenges we face when restoring proximal lesions. Basically this is the essential element in operative dentistry for assessing the correctness of the restorations of class II, III, IV. This is related to the multiple negative consequences of food retention in the proximal areas when the restorations does not properly rehabilitate the contact areas at this level (1). The entire dentomaxillary system is affected. The most frequent complications are: trauma of the marginal periodontium, with resorption of the dental papilla and the development of periodontal pockets; decrease the efficiency of the mastication function by avoiding the implicated area and using the other hemiarcade; deposits of dental plaque and carious lesions in the adjacent tooth; temporo-mandibular joint dysfunction (2).

For this reason, the design of the proximal cavities in the posterior areas of dental arcades and also the conformation modalities in order to create a perfect anatomically contact areas are extremely important. The modern preparation of class II cavities for composite materials requires a conservative approach, removing only the altered tissues strictly, without including adjacent grooves for prevention and retention and with internal rounded angles (3). 
If the dental caries is situated on the proximal surface the following types of cavities for adhesive materials(usually resin composites) can be made currently: "box preparation"; "slot cavity"; "tunnel type preparation".

In order to achieve a correct proximal restoration, it is mandatory to apply a specific conformation systems, usually called matrix. It has a metallic or plastic band and a piece that holds the matrix on the tooth (matrix retainer). The matrix acts like an opposite wall against the missing dental tooth structure, thus allowing the restoration material to be inserted and applied correctly $(4,5)$.

The role of matrix system is:

- the correct anatomical restoration of the absent dental walls;

- the correct restoration of the contact point and the configuration of the gingival embrasure;

- correct adaptation to the level of the gingival threshold, avoiding incorrect restorations, which affect or even destroy the dental papilla;

- the matrix holder and the dental wedge, produce a minimum separation of the teeth, which compensates the thickness of the matrix; once the matrix system is removed, the tooth return rapidly to the initial position and the functional correct contact point, contours and occlusion are achieved (6).

The great variety of the clinical situations that we face in restoring the proximal areas required the development of different techniques of inserting resin composites for the class II cavities. The most used techniques are: horizontal/ oblique/vertical/'U" shape/ incremental layering technique, sandwich technique, bulk fill technique, centripetal technique, successive bulk cusp build up technique and different combined techniques $(7,8)$.

\section{Case report}

In this article we present the clinical use of the UnimatrixR (TDV Dental) matrix system to restore the contact points. A patient, male, 38 years old, has presented for the treatment of multiple carious lesions on both dental arcades.
The case presented is the restoration of carious lesions in tooth 36 , located both on occlusal surface and on mesial surface(Figure 1). The occlusal cavity was previously filled with a temporary restorative material.

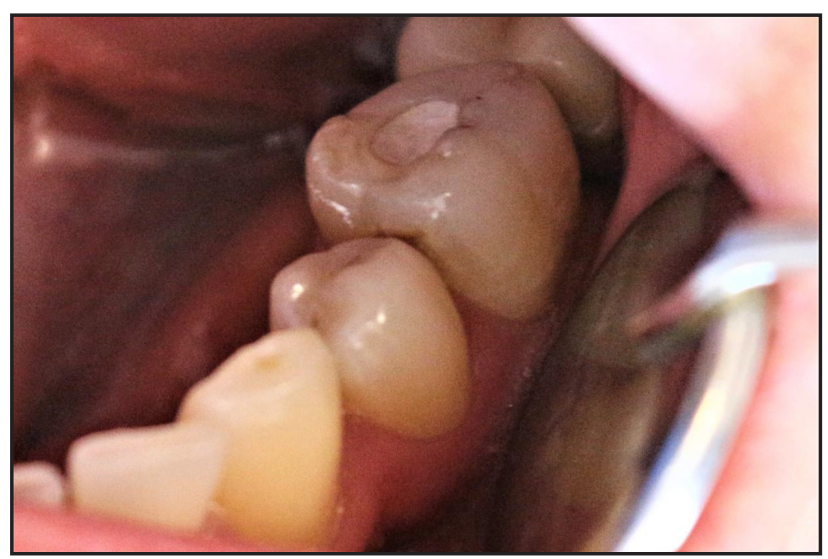

Figure 1. Carious lesion on occlusal and mesial surface in tooth 36

The treatment has started with the complete excavation and removal of all the hard dental tissues affected by carious process. A modified class II cavity has been obtained, in type of "tunnel". The contour and the shape of the cavity are done in accord with principles of modern adhesive dentistry: internal rounded angles, no extension for prevention.

The mesial marginal ridge remained intact and we decided to restore the proximal cavity first, using the centripetal technique. In order to do this, the UnimatrixR (TDV Dental) matrix system has been put in place, and stabilized with the special ring. We decided for this type of retainer system because of it's well known clinical advantages:

- the preformed sectional matrices are very thin, thus allowing to restore a tight contact point;

- the convexity of the matrix offers an anatomically restoration of proximal wall;

- the system is very easy to insert, using the specific clamp

- the comfort for the patient is higher than using classical the matrix retainer (type Tofflemire), the rigs has silicone tips which adapt perfect to proximal contour; 


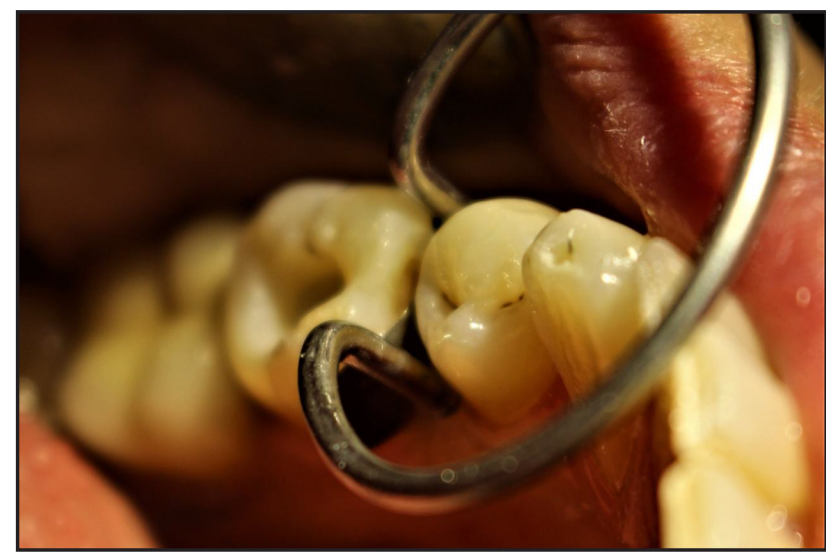

Figure 2. The UnimatrixR (TDV Dental) matrix system inserted on tooth 36

The next stage of treatment was to insert the resin composites in the proper color tooth shade we chosen previously. For this restoration we used Charisma ClassicR (Kulzer). First we isolated the tooth and then the cavity was entirely cleaned from dentin debris with $2 \%$ chlorhexidine. The total etch technique has been used, we applied $37 \%$ orthophosphoric acid both on dentin and enamel, 15 seconds for dentin and 30 seconds for enamel. (Figure 2). After completely washing of the acid the cavity has been dried and the adhesive system has been applied and light cured.

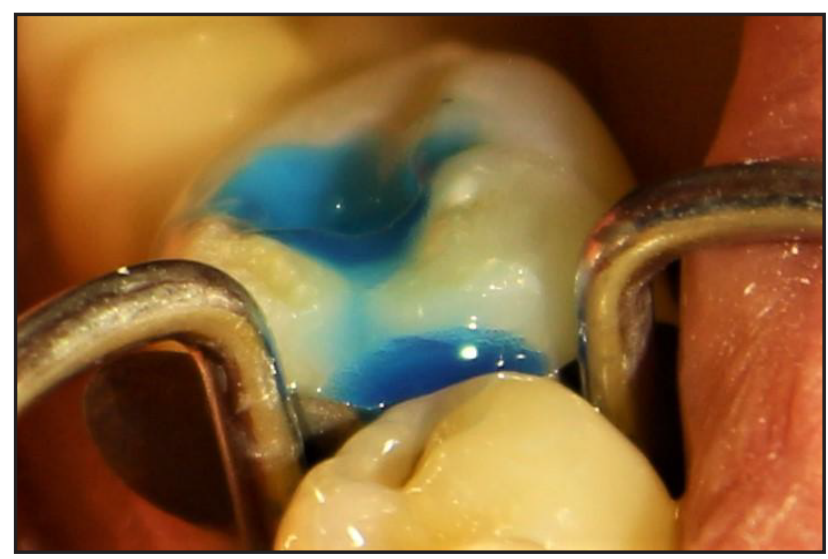

Figure 3. Total etch adhesive technique using 37\% orthophosphoric acid on tooth 36

Now the resin composite was inserted first into the mesial cavity. The mesial wall and, more important, the mesial contact point was accurate restored (Figure 3 ).

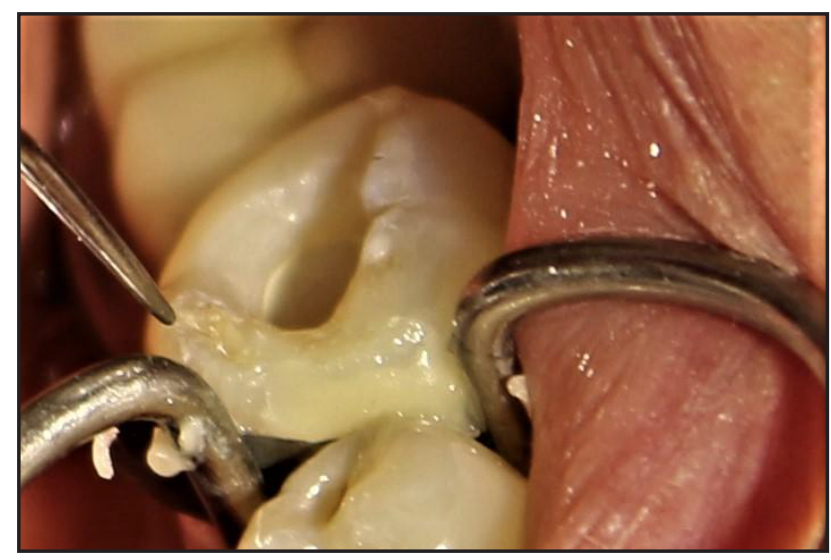

Figure 4. Reconstruction of the mesial surface and contact point on tooth 36

The next step was to restore the occlusal cavity, also with resin composites. At this point we had to correctly achieve the occlusal anatomy and morphology.

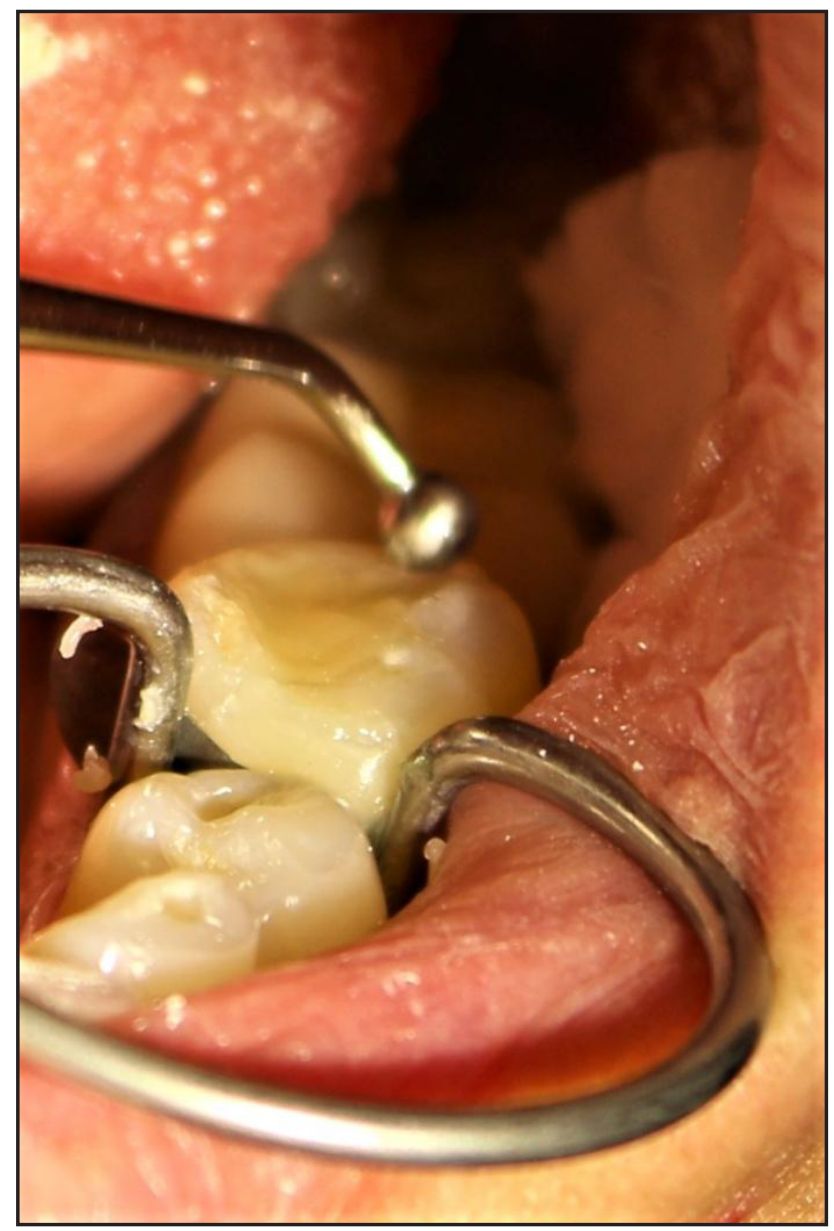

Figure 5. Restoration of occlusal cavity in tooth 36

The last stage was represented by the occlusal and functional adaptation of the restoration, using diamond burs, followed by polishing, with the help of dental polishing 
composites discs and polishing rubber cups (Figure 6).

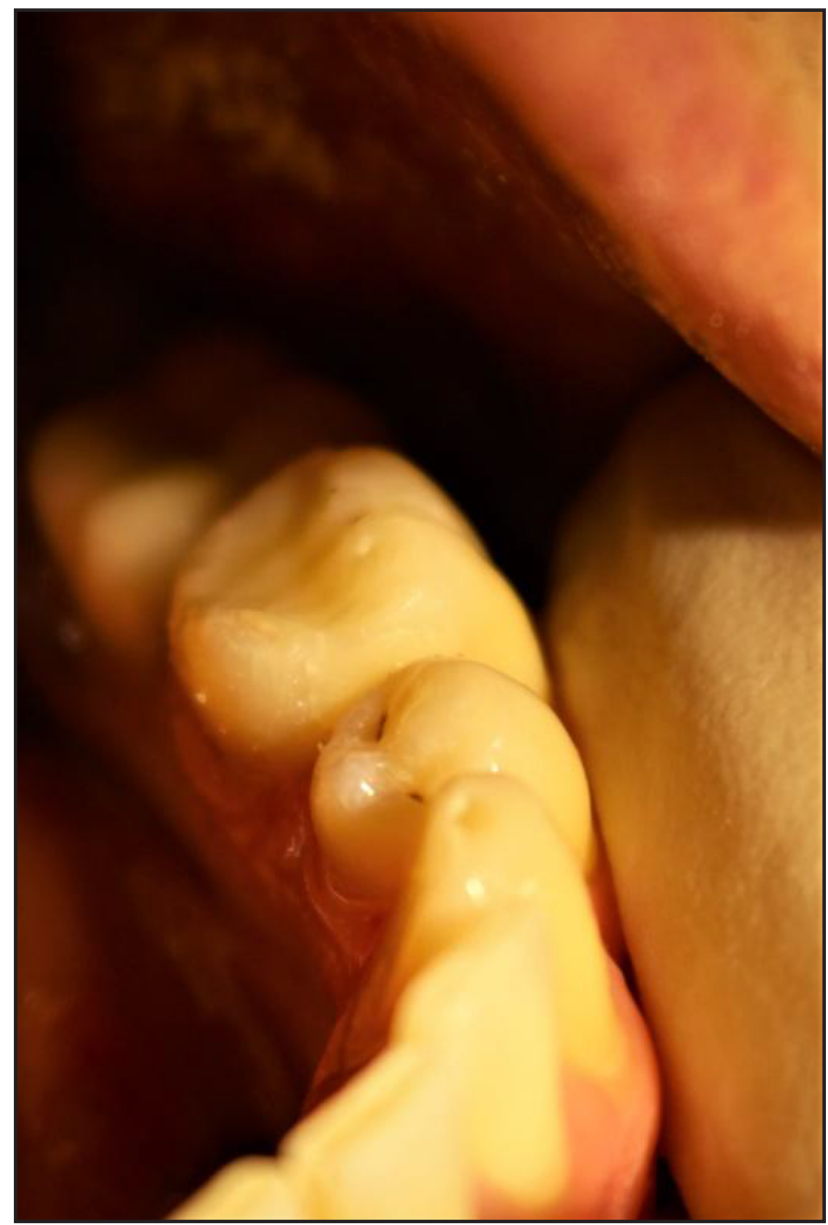

Figure 6. Final aspect of class II restoration, using UnimatrixR (TDV Dental) matrix system, in tooth 36

\section{Eonclusions}

Each working technique and each matrix system presents a number of advantages and disadvantages regarding the possibilities of correct restoration, the clinical performances, the cost price or the time required for therapeutic maneuver $(9,10)$. The major advantage of the UnimatrixR (TDV Dental) matrix system is that it allows to restore rapidly, with comfort for patient, but most of all an anatomically correct contact point and proximal contour of the missing wall.

\section{Acknowledgements:}

For this article all the authors have equal contributions.

\section{References:}

1. Calabrese M. The Importance of the Contact Point in Class Ii Restorations. Dental News. 2013 06/01;20:30-4.

2. Quadir F, Ali Abidi SY, Ahmed S. Overhanging amalgam restorations by undergraduate students. J Coll Physicians Surg Pak. 2014 Jul;24(7):485-8.

3. El-Shamy H, Saber MH, Dörfer CE, ElBadrawy W, Loomans BA. Influence of volumetric shrinkage and curing light intensity on proximal contact tightness of class II resin composite restorations: in vitro study. Oper Dent. 2012 MarApr;37(2):205-10

4. Cho SD, Browning WD, Walton KS. Clinical use of a sectional matrix and ring. Oper Dent. 2010 Sep-Oct;35(5):587-91.

5. SadafD, Ahmad MZ, Gaikwad RN, Arjumand B. Comparison of two different matrix band systems in restoring two surface cavities in posterior teeth done by senior undergraduate students at Qassim University, Saudi Arabia: A randomized controlled clinical trial. Indian J Dent Res. 2018 Jul-Aug;29(4):459-464.

6. de la Peña VA, García RP, García RP. Sectional matrix: Step-by-step directions for their clinical use. British Dental Journal. 2016 2016/01/01;220(1):11-4.

7. Chaple Gil AM. Modification of Class II technique of cavities restoration using composites. Revista Habanera de Ciencias Médicas. 2015;14(3):348-56.

8. Romero MF, Haddock F, Todd M. Combination of centripetal and successive layering techniques for a stress-reduced posterior direct composite restoration. Gen Dent. 2017 May-Jun;65(3):72-6.

9. Kampouropoulos D, Paximada C, Loukidis M, Kakaboura A. The influence of matrix type on the proximal contact in Class II resin composite restorations. Oper Dent. 2010 JulAug;35(4):454-62.

10. Wirsching E, Loomans BA, Klaiber B, Dörfer CE. Influence of matrix systems on proximal contact tightness of 2- and 3 -surface posterior composite restorations in vivo. J Dent. 2011 May;39(5):386-90. 